\title{
Criminologie
}

\section{Effets préventifs et dissuasifs : analyse d'impact d'une opération policière de prévention des cambriolages}

\section{Mathieu Charest}

Volume 36, numéro 1, printemps 2003

Police et prévention : évaluation et analyse d'impact

URI : https://id.erudit.org/iderudit/006552ar

DOI : https://doi.org/10.7202/006552ar

Aller au sommaire du numéro

\section{Éditeur(s)}

Les Presses de l'Université de Montréal

ISSN

0316-0041 (imprimé)

1492-1367 (numérique)

Découvrir la revue

Citer cet article

Charest, M. (2003). Effets préventifs et dissuasifs : analyse d'impact d'une opération policière de prévention des cambriolages. Criminologie, 36(1), 31-56. https://doi.org/10.7202/006552ar
Résumé de l'article

Cette recherche tente de développer une méthodologie évaluative destinée à départager et à comprendre la part relative des effets préventifs et dissuasifs induits par les interventions policières de prévention. Nos conclusions suggèrent que la forte baisse des cambriolages observée à la suite de la mise en place d'une opération de prévention à Boucherville (en banlieue de Montréal) est en partie imputable à une série d'arrestations ciblées de délinquants particulièrement actifs, arrestations effectuées de manière concomitante à l'implantation du projet. Plutôt que de recourir à une mesure nominale d'impact (absence/présence), nous avons utilisé une mesure continue de la force des interventions pour chaque mois. Bien que la première constitue la norme en évaluation, nos analyses démontrent qu'une mesure nominale surestime largement les effets d'une intervention. Une modélisation précise des variations chronologiques d'un processus récurrent comme la criminalité a également permis de saisir la double relation entre l'augmentation du crime, la mise en place des réponses au crime et leur impact sur les crimes subséquents. Ainsi, nous proposons un design qui considère la possibilité d'impacts différés en séquence. L'utilisation de corrélations différées a permis de déterminer le «moment optimal» où une intervention exerce son impact, tout en clarifiant les raisons pour lesquelles cette intervention exerce cet effet à tel moment plutôt qu'à tel autre.
Tous droits réservés @ Les Presses de l'Université de Montréal, 2003
Ce document est protégé par la loi sur le droit d'auteur. L’utilisation des services d'Érudit (y compris la reproduction) est assujettie à sa politique d'utilisation que vous pouvez consulter en ligne.

https://apropos.erudit.org/fr/usagers/politique-dutilisation/ 


\title{
Effets préventifs et dissuasifs: analyse d'impact d'une opération policière de prévention des cambriolages ${ }^{1}$
}

\author{
M athieu Charest \\ Candidat au doctorat \\ École de criminologie \\ Université de Montréal \\ matcharest@ hotmail.com
}

RÉSUMÉ - Cette recherche tente de développer une méthodologie évaluative destinée à départager et à comprendre la part relative des effets préventifs et dissuasifs induits par les interventions policières de prévention. Nos conclusions suggèrent que la forte baisse des cambriolages observée à la suite de la mise en place d'une opération de prévention à Boucherville (en banlieue de Montréal) est en partie imputable à une série d'arrestations ciblées de délinquants particulièrement actifs, arrestations effectuées de manière concomitante à l'implantation du projet. Plutôt que de recourir à une mesure nominale d'impact (absence/présence), nous avons utilisé une mesure continue de la force des interventions pour chaque mois. Bien que la première constitue la norme en évaluation, nos analyses démontrent qu'une mesure nominale surestime largement les effets d'une intervention. Une modélisation précise des variations chronologiques d'un processus récurrent comme la criminalité a également permis de saisir la double relation entre l'augmentation du crime, la mise en place des réponses au crime et leur impact sur les crimes subséquents. Ainsi, nous proposons un design qui considère la possibilité d'impacts différés en séquence. L'utilisation de corrélations différées a permis de déterminer le «moment optimal» où une intervention exerce son impact, tout en clarifiant les raisons pour lesquelles cette intervention exerce cet effet à tel moment plutôt qu'à tel autre.

1. L'auteur tient à remercier Pierre Tremblay pour sa précieuse collaboration et pour avoir dirigé le mémoire de maitrise qui a donné lieu à cet article. II remercie également Lyette Fusey, du Service de police de Boucherville, qui a rendu possible cette éval uation et, enfin, le Bureau d'assurance du Canada et le ministère de la Sécurité publique pour leur soutien financier. 
ABSTRACT - In this paper we assess the specific impacts of preventive and repressive actions mobilized within prevention projects initiated by police services. Findings suggest that a steep decline in burglaries following the initiation of a prevention project in a Montreal suburb was, in part, induced by a simultaneous wave of active burglar arrests. We take into account both the instantaneous and the delayed effects of preventive and repressive actions. As a result, we can identify the precise moment at which an intervention has its stronger impact. We also find that continuous measures that capture the monthly intensity of preventive resources provide a more adequate but conservative estimate of the impact of prevention programs.

\section{Introduction}

Les efforts de prévention des cambriolages résidentiels ont été dominés par deux types de mesures, la surveillance de quartier et le renforcement des cibles. Les recherches évaluatives sur l'efficacité de la surveillance ont produit des résultats décevants et souvent contradictoires. Les évaluations menées par les corps policiers s'entendent sur son efficacité, tandis que les études indépendantes suggèrent, pour la vaste majorité, une absence complète d'impact. L'efficacité semble donc varier inversement avec la qualité des designs évaluatifs (Clarke, 1995). La persistance des initiatives de surveillance s'explique en partie par les résultats prometteurs du projet de Seattle (Cirel et al., 1977), où une diminution de $61 \%$ des cambriolages avait été associée aux mesures de surveillance. Si une réévaluation du projet a montré que l'impact s'était dissipé quelques mois après l'implantation (Lindsay et M cGillis, 1986), une multitude d'initiatives du même genre ont quand même vu le jour aux États-Unis, au Canada et en Angleterre dans les années 1980, 1990 et 2000.

A Chicago, par exemple, un projet de surveillance de quartier avait comme objectif d'améliorer la cohésion sociale et les réflexes de protection des participants. Les résultats (Rosenbaum \& al., 1985) ont montré que la participation au projet n'avait eu aucun effet sur les habitudes de prévention, sur la surveillance des comportements suspects ou sur la communication d'événements inhabituels aux policiers. Aucun impact également au niveau de l'intégration sociale, des relations de bon voisinage ou de la cohésion sociale dans les quartiers. Du côté des taux de victimisation, deux des endroits ciblés ont subi une augmentation significative des cambriolages, alors qu'un seul groupe de surveillance a présenté une baisse marginalement significative de son niveau de crime. Dans la moitié des groupes, donc, le projet a provoqué ou coïncidé avec des variations qui allaient à l'inverse des hypothèses initiales. Les résultats pessimistes se 
sont accumulés au cours des années 1980 (Bennett, 1990; Skogan, 1990). Sur l'ensemble des évaluations utilisant une rigueur méthodologique minimale ${ }^{2}$, seul le projet de Seattle présente des résultats positifs. Les conclusions de Sherman et al. (1997) vont dans le même sens. Sur la base de cinq évaluations (Rosenbaum et al., 1985 ; Lindsey et M cGillis, 1986; Pate et al., 1987 ; B ennett, 1990 ; Skogan, 1990), les auteurs recommandent que le financement de ces projets soit reconsidéré.

Une deuxième catégorie de mesures propose de supprimer les occasions qui facilitent le crime en modifiant les circonstances entourant sa commission (Clarke, $1980 ; 1992 ; 1995)$. À l'aide de protections physiques, les mesures situationnelles visent à rendre le délit fastidieux, risqué ou moins profitable. L'évaluation de ces mesures a montré des résultats encourageants. En Angleterre, par exemple, une façon de réduire la vulnérabilité des résidences dans deux complexes d'habitations a été d'améliorer les serrures aux points d'entrée. Les résultats (Tilley et Webb, 1994) ont indiqué une baisse de $59 \%$ des cambriolages entre la période contrôle (avant) et la période expérimentale (après) et une baisse de $90 \%$ entre les secteurs expérimentaux et contrôles.

Toutefois, la plupart des initiatives de prévention utilisent une combinaison de mesures visant à bloquer simultanément une multitude d'opportunités criminelles (Tilley et al., 1999). Le K irkholt Burglary Prevention Project de Rochdale (Forrester et al., 1988; Forrester et al., 1990; Pease, $1991 ; 1992$; Tilley 1993) est un exemple connu des interventions dites «mixtes». L'objectif du projet de Kirkholt était de s'attaquer au problème de victimisation multiple et de diminuer le nombre global des cambriolages. Les autorités ont d'abord consenti à retirer les compteurs d'électricité (la principale cible des cambrioleurs), à la suite d'une première victimisation. D es visites sécuritaires ont également été mises en place pour améliorer les dispositifs de sécurité des domiciles cambriolés et ceux qui leurs sont immédiatement adjacents. À la suite de ces visites, les organisateurs ont formé des unités de surveillance visant à «envelopper» les résidences victimes dans une cellule de protection (le cocon) composée des six ou sept domiciles ayant une vue directe sur ces dernières. Les résultats ont montré une baisse des délits de $40 \%$, ainsi qu'une absence de victimisation multiple entre le prétest et la première année du post-test. Les auteurs conclurent que la baisse des cambrio-

2. Comparaison prétest/ post-test avec groupes contrôles, ou niveau 2 selon la codification de Sherman et al. (1997). 
lages ne pouvait être attribuable à une mesure particulière, mais bien à une combinaison de facteurs indissociables.

$M$ algré la disparité des mesures de prévention et la séquence particulière de leur implantation, les évaluateurs ont opté pour une estimation dichotomique du traitement (comparaison des délits avant et après le projet). En considérant chaque mesure comme pleinement effective du début à la fin de la période expérimentale et en éliminant toute information sur leur ordre d'implantation, il était impossible d'identifier les mesures ou les combinaisons de mesures responsables de la baisse. De plus, les auteurs ne se sont jamais intéressés à l'hypothèse selon laquelle une proportion de l'effet attribuable au projet pouvait être le résultat d'une intensification parallèle de la répression. Avec plus du quart des résidences de K irkholt cambriolées dans I'année qui a précédé le projet, il est difficile de supposer constantes des forces qui ont précisément la responsabilité de se mobiliser en contexte d'augmentation du crime.

Cet article vise à développer une méthodologie d'éval uation qui distingue et tente de comprendre ces différents effets et leurs impacts sur le crime. Nous proposons une amélioration technique des outils de recherche évaluative et une compréhension théorique des mécanismes qui opèrent une pression sur le crime. D'un point de vue technique, il est nécessaire de développer des mesures quantitatives d'intensité des projets pour dépasser leur seule évaluation qualitative ou nominale: au lieu d'évaluer l'impact des opérations de prévention sur la base de leur présence ou leur absence (prétest/ post-test), nous proposons de mesurer la «quantité» d'interventions mobilisées pour chaque mois de l'opération. II est probable qu'une évaluation considérant l'intensité des mesures comme étant optimale et constante du début à la fin de l'expérimentation surestime leurs impacts, tout en éliminant la possibilité d'estimer le quantum minimal d'intervention nécessaire pour provoquer une baisse du crime. Par ailleurs, différentes mesures de prévention peuvent opérer un effet sur le crime avec différents délais. Si on peut raisonnablement s'attendre à ce que les mesures de renforcement des cibles aient un impact immédiat sur la réduction des opportunités, et conséquemment sur les délits, il en va autrement pour les mesures de répression qui peuvent opérer sur le crime une pression différée (sur le mois suivant par exemple). L'analyse des coefficients de corrélation croisée ( «cross-correlation ») permet de raffiner les modèles d'évaluation en précisant le délai nécessaire à l'apparition d'une variation conjointe entre deux mouvements chronologiques, pour ensuite intégrer ces délais à l'intérieur d'une modélisation plus complète. 
Du point de vue théorique, la présente évaluation s'inspire des considérations de l'évaluation « réaliste» de Pawson et Tilley (1997). Contrairement aux recherches évaluatives qui tentent de contrôler (notamment par assignation aléatoire) les multiples effets contextuels sans nécessairement en connaître la nature, l'évaluation réal iste renouvelle la contribution théorique des évaluations en tenant compte des conjonctures souvent déterminantes dans la réussite ou l'échec d'une initiative de prévention. Cet article tente de voir comment une augmentation anormale de la criminalité (contexte) motive le déploiement d'efforts de contrôle préventifs et répressifs (mécanismes); il vise également à identifier ceux qui auront un impact sur les cambriolages. L'objectif est ici d'offrir une alternative à une recherche frénétique des mesures efficaces, à travers une compréhension microscopique des conjonctures et des mécanismes en cause dans une baisse de la criminalité.

\section{L'évolution des cambriolages à Boucherville}

Le projet évalué dans cet article s'est déroulé entre décembre 1998 et décembre 1999 dans la municipalité de Boucherville³, en banlieue de M ontréal. La décision de mettre en place ce projet a été motivée par une augmentation constante des cambriolages en 1997 et 1998 (figure 1).

En observant la série, on peut se demander si l'évolution des délits justifie le déploiement d'un projet de prévention. En d'autres termes, est-ce que le nombre de cambriolages commis dans les mois qui précèdent le projet s'écarte significativement des variations habituellement «tolérées» par le service de police? Pour valider l'identification «subjective» du problème de cambriolages à B oucherville, nous avons soumis la série à une analyse de discontinuité (pour une description plus détaillée, voir notamment Pettitt, 1980). C ette méthode permet précisément d'identifier la présence de changements de tendance à l'intérieur d'une série, la magnitude de ces changements et leurs périodes d'occurrence. Elle identifie les hausses ou les baisses qui se prolongent suffisamment longtemps pour s'écarter des oscillations al éatoires d'une série. Si l'iden-

3. Située en banlieue sud de M ontréal, Boucherville avait une population de 36198 habitants en 1998, répartie dans 12335 unités d'habitations (81,4\% de ces unités sont la propriété des résidents). Le revenu annuel médian par ménage se situe entre 50000 \$ et 69999 \$. Les taux de criminalité y sont relativement faibles. Toutefois, avec l'augmentation des cambriolages entre 1997 et 1998, Boucherville est passée du 8e rang (sur 10) en 1993 au $3^{e}$ rang en 1998 des municipalités ayant les plus hauts taux de cambriolages sur la rive sud de M ontréal. 
FIGURE 1

Série chronologique des cambriolages résidentiels à Boucherville, de janvier 1993 à novembre 1998 (période contrôle)

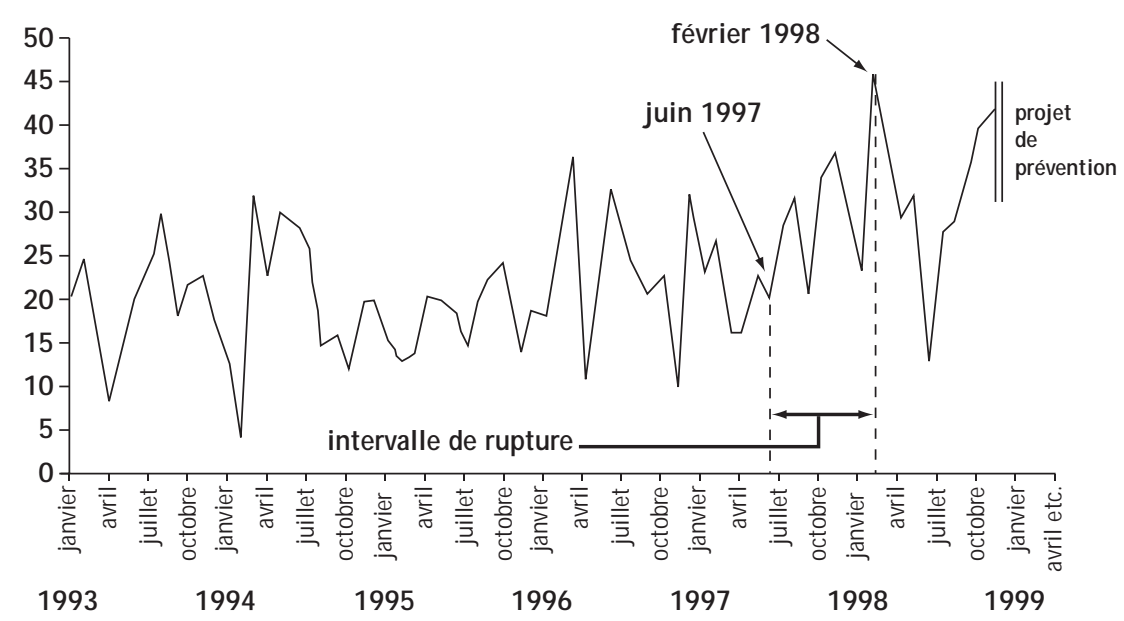

tification d'un problème criminel implique la présence d'une ou plusieurs anomalies dans la fréquence ou la gravité des crimes (Ekblom, 1988), l'analyse de discontinuité devient alors la démonstration d'un jugement auparavant arbitraire. Appliquée à la série des cambriolages, l'analyse identifie un changement de tendance qui survient entre les mois de juin 1997 et février 1998 (voir figure 1), soit dix mois avant l'implantation du projet de prévention. Durant cet intervalle, le nombre mensuel de cambriolages passe de 20,6 à 32,5, une augmentation significative de $62 \%$. Pourquoi ne pas avoir déployé le projet au plus fort de la hausse? Principal ement à cause d'une subvention qui n'est obtenue qu'en 1998. À la suite de l'obtention de la subvention, le projet a pu être mis en place à la fin de l'année 1998, au moment où une seconde hausse laissait présager un retour des cambriolages à leur niveau précédent.

En raison d'un manque de puissance des données (22 délits en moyenne par mois), nous avons utilisé un regroupement mensuel plutôt qu'hebdomadaire. M entionnons égal ement que l'année 2000 n'a pas été incluse dans le post-test, puisqu'un autre programme de prévention débutait en janvier. Les données ont été désaisonnal isées afin de limiter l'impact des perturbations cycliques et récurrentes, extrinsèques au projet de 
prévention (température, période de vacances) ${ }^{4}$, qui pourraient être responsables d'une baisse (ou d'une hausse) des délits. Cet ajustement vise principalement à ce qu'une baisse des cambriolages causée par un effet saisonnier ne soit pas créditée à tort au projet de prévention. II s'agit d'un danger plausible, puisque le premier mois de la période expérimentale (janvier) correspond à une importante baisse saisonnière des niveaux de cambriolages.

\section{Mesures de prévention et activités policières de répression}

En réponse au problème, quatre mesures de sensibilisation et trois mesures de prévention situationnelle ont été déployées. Aucune mesure n'ayant eu un impact individuel direct sur le cambriolage, nous avons procédé à deux regroupements permettant d'évaluer séparément l'impact cumulé des mesures de sensibilisation et l'impact cumulé des mesures situationnelles.

Dans le cas des mesures de sensibilisation, la variable «traitement» représente la somme mensuelle des personnes touchées par chacune des initiatives d'information ou de sensibilisation (kiosques, rencontres de groupes communautaires, dépliants d'information et articles de journaux). D ans un premier temps, un décompte des assistances a permis d'avoir le nombre précis de participants aux deux premières activités (kiosques et rencontres communautaires). $D$ ans le cas des articles de journaux et des dépliants d'information, l'estimation a été dérivée d'un sondage (Léger et Léger, $1997 ; n=912$ ) qui s'est intéressé aux taux de pénétration du journal local et des dépliants d'information. Le sondage indique que $67 \%$ des résidents lisent le journal communautaire et $66,2 \%$ avaient reçu et lu un dépliant sur des travaux municipaux projetés. Pour figurer dans ces groupes, les répondants devaient être en mesure de répondre à des questions concernant les principales informations de l'article ou du dépliant. Bien qu'approximatif, cet estimé suggère que le taux de pénétration des médias locaux est élevé. II est tout de même probable que ces deux estimés surestiment le nombre réel de personnes ayant reçu

4. La désaisonnalisation s'effectue en multipliant par 100 les valeurs de chaque mois pour ensuite les diviser par l'indice saisonnier correspondant. Par exemple, si on observe 7 cambriolages en janvier 1999, le nombre désaisonnalisé sera de 9,46 (7×100/ 73,98 - le coefficient saisonnier). Les mois d'occurrence faible seront réajustés à la hausse (et l'inverse), les variations saisonnières se trouvant ainsi contrôlées. 
et lu l'information sur les conseils de prévention. Pour limiter ce biais, nous avons opéré une transformation logarithmique des mesures de sensibilisation. Cette transformation permet de limiter le poids relatif des mesures à grand déploiement dont l'impact est incertain (articles et dépliants) tout en donnant davantage de poids aux mesures plus modestes - mais également plus intenses et informatives - qui sont susceptibles d'opérer une plus grande modification des habitudes de prévention (rencontres de citoyens).

D ans le cas des mesures situationnelles, la variable d'intensité représente le nombre cumulé des cibles (résidences) renforcées à chaque mois. Cette mesure combine le nombre de visites sécuritaires, le nombre de visites de domiciles à risques (où les policiers quadrillent le territoire pour identifier et informer les propriétaires de résidences à risque) et le nombre de personnes inscrites dans le groupe de surveillance de quartier. Le choix d'inclure la surveillance dans les mesures situationnelles rejoint la position de Tilley et al. (1999), qui suggèrent que le principe actif de cette mesure est la réduction des opportunités criminelles par une surveillance accrue. Si l'efficacité de ce type de mesure n'a pas été prouvée, il est raisonnable de penser qu'au moins les résidences des membres, qui sont pour la plupart retraités, bénéficient d'une meilleure surveillance. Finalement, comme dans le cas des mesures de sensibilisation, nous avons extrait le logarithme de chaque val eur mensuelle afin de donner plus d'importance aux initiatives dont l'impact attendu est grand (les visites sécuritaires, par exemple), mais dont l'utilisation est limitée. L'annexe 1 présente une description détaillée des effectifs mensuels de chaque groupe de mesures.

Pour contrôler l'activité policière de répression, trois indicateurs ont été inclus dans le design d'évaluation: le nombre mensuel de dossiers résolus, le nombre mensuel de mises en accusation pour cambriolages et le niveau d'activité (lambda) des groupes de cambrioleurs arrêtés dans l'année qui précède le projet et dans l'année qui l'accompagne. Contrairement aux deux premières mesures, l'estimation du lambda ne considère pas chaque arrestation ou mise en accusation comme ayant le même poids. On peut s'attendre à ce que plus un groupe a été actif dans le passé, plus l'impact de son arrestation sur la criminalité à venir sera grand. L'estimation du lambda provient de deux sources complémentaires, les dossiers d'enquêtes et les rapports de négociations entre l'enquêteur et les délinquants. Ces informations permettent d'associer un nombre de délits à un groupe de cambrioleurs, soit par modus operandi similaires soit par aveux formels en contexte d'interrogatoire ou de négo- 
ciations. Nous avons additionné l'ensemble des délits qui ont pu être formellement associés à chacun des groupes pour les trois mois qui ont précédé leurs arrestations. N ous avons ensuite ramené le total des délits de chaque groupe à la date de leurs arrestations, obtenant ainsi un estimé de l'activité antérieure des délinquants arrêtés. L'exercice permet de contrôler l'impact d'arrestations ciblées sur des délinquants particulièrement actifs et de dissocier cet impact de celui du projet de prévention. Si I'on suppose qu'une partie de l'effet dissuasif s'exerce au moment de l'arrestation ou de la mise en accusation, on possède deux points d'ancrage relativement précis qui permettront de quantifier la baisse de cambriolages associée à chaque augmentation de la pression policière.

\section{Le « design » évaluatif}

L'évaluation d'impact procède d'une analyse quasi expérimentale avec comparaison prétest et post-test du même groupe contrôle (les cambriolages), «design » auquel nous avons apporté deux modifications. Premièrement, l'ensemble des perturbations connues (effets de saisons, autorégression, opérations policières concomitantes) pouvant interférer avec l'impact des mesures de prévention ont été contrôlées directement dans nos modèles. Ce contrôle «à la source» était nécessaire, puisque nous ne pouvions présumer ces effets constants (donc s'annulant) dans les deux périodes d'analyse. Le modèle n'en est que plus puissant, puisqu'il permet de mesurer l'effet de différents mécanismes (prévention et répression) mis en place en conjoncture d'augmentation de la criminalité. Ensuite, nous avons substitué aux groupes contrôles traditionnels un «design» utilisant des "délits contrôles». Les groupes contrôles permettent de s'assurer qu'un endroit qui n'a pas bénéficié du projet de prévention n'a pas une baisse de crime équival ente aux groupes où l'intervention a eu lieu. À Boucherville, chaque citoyen devant bénéficier des mesures de prévention, il a été impossible d'isoler des secteurs contrôles exempts d'interventions. L'utilisation de délits contrôles permet toutefois d'obtenir une démonstration tout aussi convaincante. Si le projet est bien ciblé et son impact bien réel, l'effet ne devrait pas s'observer sur d'autres délits, qu'ils soient similaires ou non. N ous avons donc utilisé deux délits contrôles, I'un similaire (les cambriolages commerciaux), l'autre différent (les vols de moins de $5000 \$$ dans ou sur les véhicules). Un impact significatif sur les cambriolages commerciaux pourrait toutefois impliquer la présence d'une diffusion des bénéfices du projet vers d'autres crimes 
non ciblés a priori. Une analyse sommaire des délinquants arrêtés indique cependant que les groupes criminels sont différents pour les trois délits. II est peu probable que des mesures situationnelles visant à diminuer l'occurrence des cambriolages aient un effet sur les cambriolages commerciaux commis dans un autre secteur, par des délinquants différents, le parc industriel étant à l'écart des secteurs résidentiels.

Les données ont été analysées à l'aide de modèles AR 1 (autorégressifs d'ordre un). Contrairement à la régression linéaire, ces modèles permettent d'inclure dans l'analyse (et donc de contrôler) l'effet d'enchaînement provoqué par l'interdépendance des observations. En d'autres termes, I'analyse AR 1 prend en considération les relations qui existent entre un volume de crime au temps 1 et ce même volume au temps 2 . Appliqué aux cambriolages, un modèle autorégressif implique que le comportement des délinquants en un mois donné dépend du comportement des délinquants au cours du mois précédent (probablement parce que ce sont les mêmes et que leur fréquence de délits varie peu d'un mois à l'autre). La «mémoire» de la série est néanmoins courte, puisque les observations distancées de deux mois ne sont plus co-reliées. Le nombre de crimes d'un mois à l'autre n'est donc pas arbitraire et les processus de régulation interne de la série doivent être pris en compte (voir Charest, 2001, pour une description détaillée du modèle retenu).

\section{Mesures d'impact: comparaison prétest/ post-test}

Une première mesure d'impact consiste à vérifier si le nombre de cambriolages a effectivement diminué avec la mise en place du projet. En utilisant une période contrôle vaste (71 mois), permettant une estimation conservatrice du nombre de cambriolages avant le projet (et non simplement la hausse qui précède), les résultats montrent qu'il s'est commis en moyenne six cambriolages de moins par mois entre les deux périodes $(22,92$ comparativement à 16,92), soit une baisse de $26 \%$. Ces résultats, apparemment modestes, prennent en considération les six années qui ont précédé le projet. À titre comparatif, I'utilisation d'une période contrôle plus représentative de la situation qui prévaut avant le projet (12 mois avant) suggère plutôt une baisse de l'ordre de 49 \% (32,38 délits en 1998, contre 16,92 en 1999). La régression bivariée appuie cette dernière estimation et suggère une baisse de $45 \%(b=-14,4, p<0,01)$. Cependant, la régression estime l'impact d'une variable dichotomique $(0=$ avant, 
1 = après) sur une équation à pente positive $(0,203)$. Causée par la hausse des délits en 1997-1998, cette pente positive provoque une estimation élevée du nombre de crimes attendus dans la période expérimentale et, du même coup, surestime probablement le nombre de crimes évités grâce à l'intervention. Pour saisir l'importance de ce biais fréquent en éval uation, nous avons comparé les résultats de la régression avec ceux d'un modèle autorégressif adapté aux analyses de séries chronologiques. Les résultats (tableau 1) montrent que la force du coefficient associé au projet baisse de $40 \%$ (de-14,4 à-8,6). II est en effet peu probable que le projet ait été, à lui seul, responsable d'une diminution de $50 \%$ des délits.

TABLEAU 1

Analyse d'impact des mesures de prévention et de répression sur les cambriolages résidentiels en régression linéaire (modèle 1 ) et en modèle autorégressif (modèle 2)

\begin{tabular}{|l|c|c|c|c|c|c|}
\hline & \multicolumn{3}{|c|}{ Modèle 1 } & \multicolumn{3}{c|}{ Modèle 2 } \\
\hline & b & t ratio & sign. & b & t ratio & sign. \\
\cline { 2 - 7 } & & & & & & \\
Prévention & & & & & \\
Absence/ présence du projet & $-14,419$ & $-5,22$ &, 000 & $-8,563$ & $-2,83$ &, 006 \\
\hline Répression & & & & & & \\
Nombre de résolutions & $-0,012$ & $-0,64$ &, 949 & 0,028 & 0,14 &, 888 \\
Nombre d'accusations & 0,665 & 2,74 &, 008 & 0,868 & 3,95 &, 000 \\
\hline Contrôles & & & & & & \\
Période (tendance générale) & 0,193 & 3,49 &, 001 & - & - & - \\
\hline AR1 & - & - & - & 0,350 & 2,95 &, 004 \\
Constante & 14,037 & 6,06 &, 000 & 21,678 & 14,61 &, 000 \\
Durbin-Watson & 1,497 & & & & & \\
N & 72 & & & 72 & & \\
R carré & 0,410 & & & 0,307 & & \\
\hline
\end{tabular}

Les premiers indicateurs de l'activité policière (le nombre de résolutions et le nombre d'accusations) n'ont pas d'impact sur le mouvement des cambriolages. Le nombre d'accusations est relié positivement au nombre de cambriolages. Intuitivement, une relation négative entre ces deux variables était attendue. Un coefficient positif suggère une relation causale inverse, où l'augmentation du nombre de cambriolages permet un plus grand nombre d'accusations. On ne peut toutefois pas évacuer la possibilité que l'effet dissuasif des mises en accusation se précise avec les délits du mois suivant, phénomène tout aussi plausible pour les résolutions. 
La première estimation des impacts du projet soulève deux lacunes de notre «design » d'évaluation. La première relève de la nature dichotomique des variables d'impact, la seconde, de la présence possible de causalités différées.

\section{Intensité variable des mesures de prévention}

Pour remédier à la nature dichotomique de la variable d'impact, nous avons utilisé une mesure continue qui tient compte à la fois des variations dans l'intensité des mesures et des variations dans les catégories de mesures utilisées pour chaque mois. Comme nous l'avons mentionné, ces mesures utilisent la somme des personnes touchées par chacune des mesures de sensibilisation et la somme des résidences renforcées par les mesures de prévention situationnelle. La précision de ces indicateurs est nettement supérieure à une mesure dichotomique, qui suppose une intensité optimale et constante de l'opération.

\section{TABLEA U 2}

Analyse d'impact des mesures de prévention et de répression utilisant une mesure dichotomique de traitement (modèle 2) et une mesure continue d'intensité (modèle 3 ) de cambriolages

\begin{tabular}{|l|c|c|c|c|c|c|}
\hline & \multicolumn{3}{|c|}{ Modèle 2 } & \multicolumn{3}{c|}{ Modèle 3 } \\
\hline & b & t ratio & sign. & b & t ratio & sign. \\
\cline { 2 - 7 } Prévention & & & & & & \\
$\begin{array}{l}\text { Absence / présence du projet } \\
\text { Mesures situationnelles } \\
\text { (log10) }\end{array}$ & $-8,563$ & $-2,83$ &, 006 & - & - & - \\
$\begin{array}{l}\text { Mesures d'information } \\
\text { (log10) }\end{array}$ & - & - & - & 1,513 & 1,31 &, 194 \\
\hline Répression & & & & & & \\
Nombre de résolutions & 0,028 & 0,14 &, 888 & 0,068 & 0,33 &, 744 \\
Nombre d'accusations & 0,868 & 3,95 &, 000 & 0,845 & 3,86 &, 000 \\
\hline Contrôles & & & & & & \\
AR1 & 0,350 & 2,95 &, 004 & 0,353 & 2,94 &, 005 \\
Constante & 21,678 & 14,61 &, 000 & 21,517 & 14,34 &, 000 \\
N & 72 & & & 72 & & \\
R carré & 0,307 & & & 0,321 & & \\
\hline
\end{tabular}


En dissociant les catégories de mesures et en tenant compte de leurs variations d'intensité (tableau 2, modèle 3), on remarque que seules les mesures situationnelles ont eu un impact significatif sur la diminution des délits $(b=-6,77, p<0,01)$. En fait, cette variable représente le seul facteur qui covarie négativement avec le nombre de cambriolages. La force du coefficient diminue de $21 \%$, confirmant les risques de surestimation associés à la variable polarisée du modèle 2 . Le «principe actif » du programme, les mesures situationnelles dans ce cas-ci, n'est pas constant d'un mois à l'autre, alors que la baisse des cambriolages l'est. La relation entre les deux séries chronologiques diminue conséquemment. Le coefficient positif associé aux mesures de sensibilisation est contraire aux attentes de l'équipe de prévention, qui y voyait un levier prometteur dans le changement des habitudes de prévention. On peut toutefois penser qu'une modification des habitudes, si elle est observable, ne sera pas instantanée. II est quand même intéressant de noter que, dans son ensemble, le programme de prévention induit deux mouvements opposés.

FIGURE 2

\section{Variations chronologiques de l'intensité des mesures de prévention situationnelle} et des cambriolages résidentiels (période expérimentale)

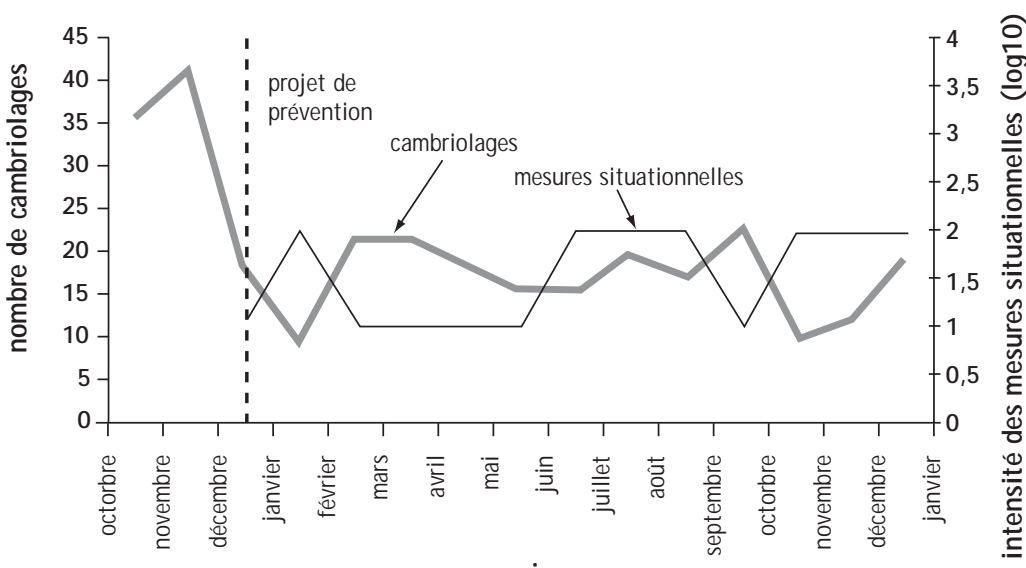

mois

Les résultats du modèle 3 impliquent également le rejet de l'hypothèse d'un artefact méthodologique, qui aurait fait correspondre a posteriori le début du projet avec la baisse des délits. Ce danger n'est pas sans importance, puisqu'en observant la chute des cambriolages, en décembre 1998 et janvier 1999, l'équipe de prévention aurait pu (motivée par une obligation de résultats) déplacer légèrement la période expérimentale pour 
qu'elle corresponde parfaitement au début de la chute. Un suivi précis de I'intensité des mesures permet d'éliminer ce doute. Pour mieux apprécier les relations du modèle 3, nous avons superposé, pour la période expérimentale, la série des mesures situationnelles et la série des cambriolages.

La figure 2 montre que l'initiation des mesures situationnelles débute après le premier mois de baisse des délits. Avec l'arrivée des mesures situationnelles, les crimes poursuivent leur descente sous les moyennes antérieures. Cette baisse correspond exactement à la première pointe d'intensité des mesures en janvier 1999. La rotation avec les mesures de sensibilisation fait ensuite baisser le nombre d'opérations situationnelles et, au même moment, les délits remontent. La deuxième pointe qui survient aux mois de juin, juillet et août s'insère dans ce qui semble être un mouvement de hausse des délits qui débute timidement en juin. II est possible que les mesures de prévention situationnelle, même si elles ne parviennent pas à faire baisser les délits durant l'été, opposent un frein suffisant pour limiter le retour progressif des délits à leurs niveaux précédents (les coefficients saisonniers prévoient des hausses durant ces trois mois). Les activités policières de répression étant en «arrêt» à cette période ( 4 dossiers résolus et 2 mises en accusation pour les trois mois), on peut supposer que le poids des mesures situationnelles est un des principaux facteurs explicatifs de cette régulation. La hausse instantanée qui survient au moment où les mesures situationnelles se relâchent, en septembre 1999, bonifie cette hypothèse. À partir de ce moment, la covariation entre les deux phénomènes est particulièrement étroite. Le retour d'intensité en octobre fera baisser le nombre de cambriolages pour un temps. Ensuite, même si l'intensité se prolonge, le nombre de délits augmente.

\section{Causalité différée: direction et persistance des effets}

Tout impact n'étant pas instantané, il est possible d'observer des effets causals différés, et ce, même quand le traitement est implanté de manière homogène (Cook et Campbell, 1979: 227). Ces effets différés ne posent pas problème lorsqu'un appui théorique permet de prédire le délai entre le traitement et l'effet attendu. La plupart du temps, par contre, ces théories n'existent pas et l'évaluation d'impact, sans considérer la présence de délais possibles, n'identifiera pas un ensemble de mouvements causals plus subtils.

En évaluant les actions policières et préventives mises en place pour contrer le crime, on peut s'attendre à des interactions croisées. D 'un côté, 
les mouvements de la criminalité justifient l'établissement d'une réponse préventive ou répressive. De l'autre, ces opérations, espère-t-on, auront un effet régulateur sur le mouvement de la criminalité. Les diagrammes de corrélations différées permettent précisément d'identifier ces interactions. Cet outil exploratoire permet également de spécifier le délai précis nécessaire à l'actualisation de l'impact pour chaque combinaison de variables et d'intégrer ce délai dans un modèle statistique plus complet. Cette procédure permet finalement d'estimer la persistance de l'effet préventif ou répressif une fois les mesures interrompues. La figure 3 présente le diagramme de corrélations différées pour les mesures de prévention.

La plus forte corrélation entre les mesures situationnelles et les cambriolages apparaît en temps 0 , sans délai apparent (diagramme de gauche). S'il y avait eu effet différé, la corrélation aurait été plus puissante en temps 1 qu'en temps 0 . N éanmoins, le coefficient au délai 1 est toujours significatif, ce qui signifie que les pointes d'intensité des mesures de prévention situationnelle sont toujours reliées à une baisse récurrente des crimes au mois suivant. L'effet instantané des mesures situationnelles est conséquent avec les principes de la théorie des opportunités. Le renforcement des cibles devrait avoir un impact immédiat sur la réduction des opportunités de cambriolages.

Contrairement à ce que suggérait le dernier modèle, la figure 3 (diagramme de droite) montre que l'effet des mesures d'information est relié négativement au nombre de cambriolages. Le simple fait que ces mesures aient été implantées au moment où les crimes augmentaient suffit à masquer leur impact bivarié au même moment. II faut attendre deux mois avant de voir une corrélation significative entre les deux séries. Théoriquement, il est difficile d'expliquer pourquoi les délits baissent deux mois après les vagues de sensibilisation. II est possible que l'amélioration des réflexes de prévention relève d'un processus cumulatif plutôt que ponctuel. II est possible que le seuil critique de personnes ayant amélioré la sécurité de leur domicile à cause de la campagne de sensibilisation ne soit atteint qu'après deux mois, suivant un processus additif. Si les résultats sont intéressants en bivarié, I'hypothèse du cumul devra être vérifiée dans un modèle multivarié.

Jusqu'à présent, les analyses ont montré que la résolution des dossiers n'a aucun effet sur le nombre de délits commis. En temps réel, la relation entre les deux séries est nulle. Le diagramme de corrélations (non présenté) indique toutefois la présence d'une double relation (l'une positive, l'autre négative), entre le volume des crimes et la résolution des 


\section{FI GURE 3}

Diagrammes des corrélations différées entre les mesures de prévention situationnelle et le nombre mensuel de cambriolages (diagramme de gauche) et entre les mesures de sensibilisation et le nombre mensuel de cambriolages résidentiels (diagramme de droite)

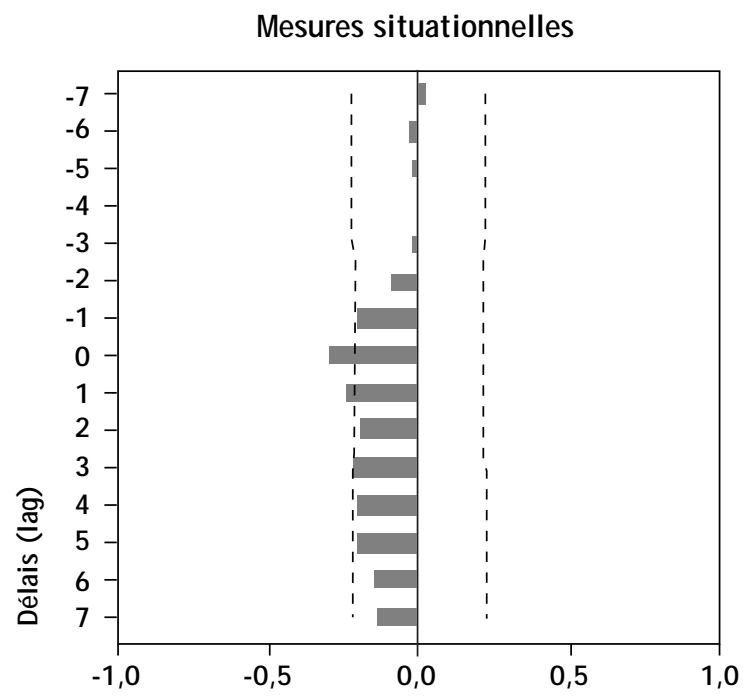

Mesures de sensibilisation

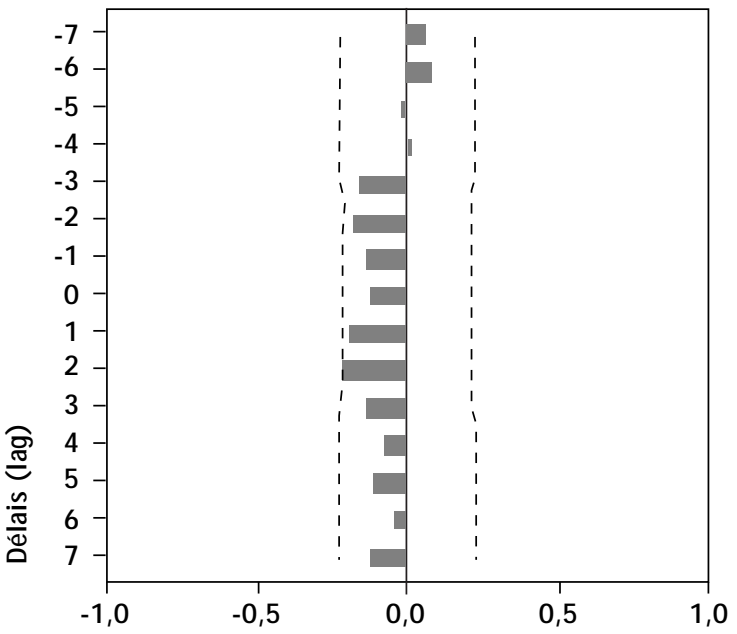


dossiers. D'une part, on observe que le nombre de délits commis au mois $x$ sont corrélés positivement avec le nombre de dossiers solutionnés au mois $x+3$. Plus il y a de crimes commis, plus il y aura de résolutions dans les mois qui suivent, avec une pointe au troisième mois. $\mathrm{Ce}$ «retard » peut provenir du fait qu'un seul enquêteur est affecté aux cambriolages à Boucherville. D'autre part, le délai des résolutions semble se répercuter sur la deuxième section de la chaîne causale. II faut en effet attendre trois mois avant que l'augmentation des résolutions soit reliée négativement au nombre de crimes. En contexte d'évaluation, la capacité de saisir adéquatement l'impact des résolutions sur les crimes futurs nous intéresse plus particulièrement. En fait, le bureau des enquêtes criminelles était convaincu que la hausse des résolutions était la véritable cause de la chute des cambriolages au début de l'année 1999. Or, si c'est le cas, notre modélisation n'a pas été en mesure de bien saisir cet impact. II est donc nécessaire de différer la correspondance des séries de trois mois.

Finalement, le nombre mensuel d'accusations était la dernière variable pour laquelle des problèmes de directions causales auraient pu se poser. La corrélation différée vient dissiper ce doute. L'augmentation du nombre d'accusations en un mois $\mathbf{t}$ ' 'a pas d'effet sur la criminalité future. La relation la plus forte apparaît en temps réel (délai 0 ), moment où les deux phénomènes sont reliés positivement. La menace des poursuites judiciaires $n$ 'arriverait pas à dissuader les délinquants de ralentir leurs activités, soit en raison des peines auxquelles ils font face, soit à cause du trop petit nombre de cambrioleurs visés par ces poursuites. II est égal ement possible que la menace pénale s'exerce bien avant la mise en accusation, au moment de l'arrestation et de l'interrogatoire, par exemple.

En résumé, deux modifications ont été effectuées au modèle précédent, une sur la variable des mesures d'information (délai de 2 mois), l'autre sur les nombres mensuels de résolutions (délai de 3 mois). Les deux autres variables, les mesures situationnelles et les mises en accusation, seront incluses en temps réel. Le tableau 3 présente les résultats.

Premier changement: toutes les variables, hormis les mises en accusation, présentent des coefficients négatifs. Cette meilleure estimation des effets concomitants s'accompagne également d'une diminution considérable de l'impact des mesures situationnelles (- $32 \%)$. C ette baisse est attribuable, en partie, à l'effet dissuasif des résolutions, qui est significatif $(-0,304, p<0,05)$. Dans le cas des mesures de sensibilisation, leur impact sur le crime est beaucoup trop incertain pour être pris en compte. 
TABLEA U 3

Analyse d'impact des mesures de prévention et de répression sur les cambriolages résidentiels, incluant les mesures continues d'intensité et les effets différés (modèle 4)

\begin{tabular}{|l|c|c|c|c|c|c|c|}
\hline & \multicolumn{4}{|c|}{ Modèle 3 } & \multicolumn{5}{c|}{ Modèle 4 } \\
\hline & b & t ratio & sign. & b & t ratio & sign. & lag \\
\cline { 2 - 8 } & & & & & & & \\
Prévention & $-6,772$ & $-3,06$ &, 003 & $-4,579$ & $-2,16$ &, 034 & 0 \\
Mesures situationnelles & 1,513 & 1,31 &, 194 & $-0,823$ & $-0,76$ &, 453 & 2 \\
Répression d'information & & & & & & & \\
Nombre de résolutions & 0,068 & 0,33 &, 744 & $-0,304$ & $-2,58$ &, 012 & 3 \\
Nombre d'accusations & 0,845 & 3,86 &, 000 & 0,812 & 3,87 &, 000 & 0 \\
Contrôles & & & & & & & \\
AR1 & 0,353 & 2,94 &, 005 & 0,386 & 3,32 &, 002 & - \\
Constante & 21,517 & 14,34 &, 000 & 22,769 & 15,33 &, 000 & - \\
N & 72 & & & 72 & & & \\
R carré & 0,321 & & & 0,377 & & & \\
\hline
\end{tabular}

En comparant la valeur prédite au dernier mois de la période contrôle $(35)^{5}$ avec le nombre moyen de crimes durant la période expérimentale (17), nous estimons qu'en moyenne 19 délits de moins ont été commis (par mois) durant la période expérimentale. Les dispositifs de prévention situationnelle auraient donc contribué à $24 \%(4,57 / 19)$ de la baisse totale des cambriolages.

\section{Les délits contrôles}

Le modèle final a ensuite été appliqué à deux délits non ciblés par le projet. Cette démonstration par la négative permet de s'assurer que les impacts de l'opération de prévention ne s'observent que sur le délit ciblé au départ. Elle permet également de voir si la mise en place du projet s'accompagne d'un déplacement de cibles (des cambriolages résidentiels aux cambriolages commerciaux, par exemple).

5. L'équation fixe la constante à 22,8 crimes, ce qui représente le niveau initial de crime pour la série. Pour estimer le niveau de crime du mois qui précède le projet de prévention, on multiplie le nombre d'observations de la période contrôle (59) par le coefficient de pente de la série $(0,207)$. On obtient alors la progression des cambriolages pour la période expérimentale (12,21 délits). En additionnant ce nombre à la constante initiale, l'équation estime à 35 le nombre prédit de cambriolages au cours du mois qui précède le projet. 
Le tableau 4 suggère que les mesures situationnelles ont eu un impact sur le cambriolage résidentiel seulement. Aucun effet significatif n'est observable pour les cambriolages commerciaux et pour les vols de moins de $5000 \$$ dans ou sur les véhicules. Ces résultats sont conséquents avec l'analyse des cambriolages commerciaux qui avait montré que ces deux types de délits étaient l'œuvre de délinquants différents. $0 n$ remarque également que les mesures de prévention n'ont pas été accompagnées d'une augmentation significative du nombre de cambriolages commerciaux, ce qui limite l'hypothèse d'un déplacement de cibles. Les cambrioleurs résidentiels ne semblent pas être prêts à s'impliquer dans les vols commis dans des commerces qui demandent une expertise et des moyens d'action différents.

\section{Lambda des groupes arrêtés et dissuasion cumulée}

Finalement, une analyse exploratoire utilisant le lambda des groupes arrêtés a été envisagée, à la suite de quelques interrogations sur la qualité de nos indicateurs de répression policière. Ces indicateurs ont le désavantage de considérer toutes les arrestations comme équival entes. L'utilisation d'une mesure de dissuasion plus sensible vise à vérifier deux hypothèses relatives à l'impact des opérations policières de répression. N ous pensons premièrement que l'efficacité réelle des opérations policières réside dans une capacité à neutral iser les délinquants les plus prolifiques. L'arrestation des principaux cambrioleurs, à la fin de l'année 1998, pourrait être une des causes les plus importantes de la chute des délits au début de l'année 1999. Deuxièmement, nous croyons qu'une série de frappes policières qui arrivent à cumuler des arrestations à un rythme rapide dans un court laps de temps (de 2 à 4 mois), auraient un impact plus puissant et prolongé que ces mêmes arrestations dispersées sur une plus longue période. Le cumul dissuasif de ces arrestations permettrait de déstabiliser une fraction critique des délinquants les plus actifs, provoquant ainsi un vacuum temporaire de l'activité criminelle.

Le défi était d'estimer le lambda des groupes criminels et, ensuite, d'opérationnaliser l'effet de dissuasion cumulée. Nous l'avons mentionné, le lambda a été estimé à partir des négociations entre l'enquêteur et les cambrioleurs. En ce qui concerne l'effet de cumul, nous avons utilisé un calcul de moyennes mobiles qui permet de pondérer le poids d'une arrestation en considérant la séquence dans laquelle elle s'introduit. Les arrestations qui s'insèrent à l'intérieur d'une séquence de répression élevée seront donc pondérées à la hausse. Les moyennes mobiles permettent 
TABLEAU 4

Analyse des impacts des mesures de prévention et de répression sur les cambriolages résidentiels,

les cambriolages commeriaux et sur les vols de moins de 5000\$ dans/ sur des véhiales (1994-1999)

\begin{tabular}{|c|c|c|c|c|c|c|c|c|c|}
\hline & \multicolumn{3}{|c|}{$\begin{array}{c}\text { Cambriolages résiclentiels } \\
\text { ARIMA }(1,0,0)\end{array}$} & \multicolumn{3}{|c|}{$\begin{array}{c}\text { Cambriolages commerciaux } \\
\text { ARIMA }(1,0,0)\end{array}$} & \multicolumn{3}{|c|}{$\begin{array}{c}\text { Vols de moins de } 5000 \$ \text { dans/ sur } \\
\text { véhiales ARIMA }(0,0,1)\end{array}$} \\
\hline & $\mathbf{b}$ & t ratio & sign. & $\mathbf{b}$ & t ratio & sign. & $\mathbf{b}$ & t ratio & sign. \\
\hline \multicolumn{10}{|l|}{ Prévention } \\
\hline Mesures situationnelles & $-4,579$ & $-2,16$ &, 034 & 0,035 & 0,02 & ,983 & 1,309 & 0,52 & ,605 \\
\hline Mesures d'information & $-0,823$ & $-0,76$ & ,453 & 1,044 & 1,34 & , 186 & 0,412 & 0,30 & ,764 \\
\hline \multicolumn{10}{|l|}{ Répression } \\
\hline Nombre de résolutions & $-0,304$ & $-2,58$ & ,012 & $-0,010$ & $-0,04$ & ,968 & 0,838 & 3,45 & ,001 \\
\hline Nombre d'accusations & 0,812 & 3,87 &, 000 & $-0,086$ & $-0,23$ & ,815 & $-0,113$ & $-0,10$ & ,918 \\
\hline \multicolumn{10}{|l|}{ Contrôles } \\
\hline ARl & 0,386 & 3,32 &, 002 & 0,420 & 3,68 &, 000 & - & - & - \\
\hline MA1 & - & 一 & 一 & 一 & 一 & 一 & $-0,369$ & $-3,13$ &, 003 \\
\hline Constante & 22,769 & 15,33 & , 000 & 10,963 & 10,29 &, 000 & 15,267 & 10,49 & , 000 \\
\hline$N$ & 72 & & & 82 & & & 72 & & \\
\hline R carré & 0,377 & & & 0,171 & & & 0,238 & & \\
\hline
\end{tabular}


également de tenir compte de l'effet récurrent des frappes policières. N ous avons fixé la longueur de la mobilité à trois mois. Au-delà de cette période, il est incertain qu'une arrestation ait un impact sur le nombre de cambriolages en cours.

Le tableau 5 présente les résultats de l'analyse exploratoire pour 1998 et 1999. N ous qualifions cet exercice d'exploratoire, puisque le nombre d'observations ne respecte pas les postulats des modèles chronologiques (le nombre d'observations doit être supérieur à 50). Toutefois, étant donné la forte autocorrélation des observations adjacentes, nous avons préféré cette méthode à la régression linéaire.

Le modèle 5 a reprend les mêmes améliorations qui ont été apportées aux modèles précédents. En ne considérant que les 24 derniers mois de la série, il n'a toutefois pas été possible d'identifier des effets de délais qui auraient mérité que l'on décale l'un ou l'autre des indicateurs de répression policière. Seule la variable «mesures de sensibilisation » a été différée. La principale différence entre les deux modèles se situe au niveau de la force prédictive des indicateurs de répression policière. $D$ ans le premier modèle, l'augmentation des résolutions ou des mises en accusation n'a pas d'impact significatif sur les cambriolages au même mois. En considérant le niveau d'activité des groupes arrêtés en 1998 et en 1999 et l'effet de cumul de ces arrestations (modèle $5 b$ ), on remarque que le coefficient associé à l'activité policière concomitante devient beaucoup plus puissant $(b=-3,697)$ et sensiblement plus robuste $(p=0,11)$. Ces effets conjoints présentent une piste de solution particulièrement intéressante dans l'explication de la baisse des cambriolages survenue au début du programme de prévention. Les variables relatives au nombre de résolutions et aux mises en accusation ne semblent pas être en mesure d'isoler cet effet, ni de tenir compte de la puissance cumulée des arrestations successives.

Limitée par l'information que nous possédions, l'estimation du poids des groupes et de l'effet de cumul présente évidemment certaines lacunes qu'il aurait été possible de contourner avec une information précise du lambda de chaque délinquant. Cette lacune soulève l'importance de développer des méthodes qui rendront possible cette estimation. D u côté des mesures de prévention, l'utilisation d'une période contrôle plus courte semble accentuer la rupture qui survient entre les deux segments d'analyse (contrôle par opposition à expérimentale). L'augmentation du coefficient (b) attribuée aux mesures situationnelles provient en partie de l'estimation initiale des cambriolages qui est révisée à la hausse, puisque les délits de 1998 étaient beaucoup plus élevés que ceux de la période contrôle précédente (1994 à 1998). II est tout de même intéressant de 
TABLEAU 5

Analyse drimpact des mesures de prévention et de répression sur les cambriolages résidentiels,

induant l'estimation cu lambda des groupes criminels arrêtés et l'effet de amul des arrestations (modèle 5b)

\begin{tabular}{|c|c|c|c|c|c|c|c|c|}
\hline & \multicolumn{4}{|c|}{ Modèle 5a } & \multicolumn{4}{|c|}{ Modèle 5b } \\
\hline \multirow[b]{2}{*}{ Prévention } & $\mathbf{b}$ & t ratio & $\operatorname{sign}$. & lag & $\mathbf{b}$ & t ratio & sign. & lag \\
\hline & & & & & & & & \\
\hline Mesures situationnelles & $-7,883$ & $-3,73$ &, 002 & 0 & $-7,532$ & $-3,05$ & ,007 & 0 \\
\hline Mesures d'information & 0,234 & 0,22 & ,830 & 1 & $-0,990$ & $-0,77$ & ,454 & 1 \\
\hline Répression & & & & & & & & \\
\hline Nonbre de résolutions & $-0,189$ & $-0,64$ &, 530 & 0 & - & - & - & - \\
\hline Nombre d'accusations & 0,624 & 2,40 & ,027 & o & - & - & - & - \\
\hline Groupes actifs arrêtés & - & - & - & - & $-3,697$ & $-1,65$ &, 116 & 0 \\
\hline Contrôles & & & & & & & & \\
\hline$A R 1$ & 0,284 & 1,29 & ,215 & & 0,149 & 0,64 &, 530 & \\
\hline Constante & 28,126 & 9,11 & , 000 & & 32,250 & 14,14 & , 000 & \\
\hline$N$ & 24 & & & & 24 & & & \\
\hline R carré & 0,578 & & & & 0,537 & & & \\
\hline
\end{tabular}


noter que, même en améliorant la mesure de répression policière, l'effet des mesures de prévention situationnelle conserve sa force (- 7,88 par opposition à - 7,53). Les mesures situationnelles demeurent le principal facteur ayant contribué à la baisse des cambriolages en 1999.

\section{Conclusion}

II est possible que la motivation d'un corps de police à mettre sur pied un programme de prévention situationnelle l'incite également à accentuer et cibler ses efforts répressifs. L'objectif de cette recherche a été de vérifier les mérites d'une telle hypothèse. $N$ os conclusions suggèrent que la baisse des cambriolages observée à la suite de la mise en place d'un projet de prévention est en partie imputable à une série d'arrestations ciblées de délinquants particulièrement actifs qui se sont produites de manière «concomitantes» à la mise en place du projet de prévention. II n'en reste pas moins que les mesures de prévention situationnelle ont été efficaces. L'estimation la plus conservatrice de l'ordre de grandeur de ces interventions indique que ces mesures ont contribué à $24 \%$ de la baisse observée. Cette estimation tient compte à la fois de l'intensité variable des mesures, des fluctuations saisonnières de la criminalité, des effets attendus de "retour statistique à la moyenne», de la tendance de la criminalité dans le site d'analyse (sur une période de quatre ans) et de l'impact dissuasif induit parallèlement par l'arrestation de délinquants particulièrement actifs. Cette analyse est d'autant plus convaincante qu'il a été possible de démontrer que ces opérations de prévention n'ont eu aucun impact sur des types de criminalité qu'elles ne ciblaient pas au départ et pour lesquels il n'existait aucune raison particulière de penser qu'elles pourraient a priori les influencer.

U ne modélisation précise des variations chronologiques d'un processus récurrent comme la criminal ité a également pour avantage non seulement d'offrir une appréciation plus réal iste des interventions de prévention, mais également une meilleure compréhension des efforts mobilisés en contexte d'augmentation de la criminalité et de leurs impacts immédiats ou différés sur le crime. Ainsi, on évalue mieux l'impact des opérations de sensibilisation ou d'information des projets de prévention en évaluant leurs effets différés sur les niveaux de cambriolage (deux mois plus tard); on évalue mieux l'impact dissuasif des enquêtes criminelles «élucidées» en évaluant leurs effets différés sur les niveaux de cambriolage trois mois plus tard qu'en évaluant leur impact immédiat. Les diagrammes de corrélations différées constituent, de ce point de vue, un 
outil analytique précieux non seulement pour déterminer le «moment optimal » où une intervention de prévention exercerait son impact attendu, mais pour clarifier les raisons pour lesquelles cette intervention devrait exercer cet effet à tel moment plutôt qu'à tel autre. Répondre à de telles questions impose aux préventionnistes non seulement de statuer sur le degré d'efficacité des ressources mobilisées, mais également, et surtout, de comprendre les raisons éventuelles de cette efficacité présumée.

\section{Références}

Bennett, T. (1990). Evaluating N eighbourhood W atch. Cambridge: Gower, Cambridge University, Studies in Criminology.

Cirel, P., Evans, P., M cGillis, D., \& Weitcomb, D. (1977). An ex emplary project: Community Crime Prevention Programme, Seattle, W ashington. Washington D.C. : N ational Institute of Law Enforcement and C rime Justice.

Charest, M. (2001). Effets préventifs et effets dissuasifs: analyse quasiex périmentale d'une opération policière de prévention des cambriolages. M émoire de maîtrise, École de criminologie, U niversité de M ontréal.

Clarke, R.V. (1980). Situational crime prevention: theory and practice. The British Journal of Criminology, 20 (2), 136-147.

Clarke, R.V. (1992). Situational crime prevention. Successful case studies. N ew York: Harrow and Heston.

Clarke, R.V. (1995). Situational crime prevention. In M. Tonry \& D.P. Farrington (eds), Building a safer society: Strategic approaches to crime prevention. Crime and Justice: A Review of Research, 19, 91-150. Chicago: U niversity of Chicago Press.

Cook, T.D., \& Campbell, D.T. (1979). Q uasi-experimentation: design and analysis issues for field settings. C hicago : R and- $\mathrm{M} \mathrm{CN}$ ally.

Ekblom, P. (1988). G etting the best out of crime analysis. C rime Prevention Unit Paper 10. Londres: H ome 0 ffice.

Forrester, D., Chatterton, M., \& Pease, K. (1988). T he K irkholt Burglary Prevention Project: Rochdale. Crime Prevention U nit Paper no. 13. Londres: Home 0 ffice.

Forrester, D., Frenz, S., O 'C onnell, M ., \& Pease, K . (1990). The K irkholt Burglary Prevention Project: phase II. Crime Prevention Unit Paper no. 23. Londres: Home $O$ ffice. 
Léger \& Léger (1997). Profil socio-démographique des citoyens interrogés. Ville de Boucherville.

Lindsay, B., \& M cGillis, D. (1986). Citywide community crime prevention : an assessment of the Seattle program. In D.P. R osenbaum (ed), Community crime prevention: does it work? (19-44). Beverly Hills: Sage Publications.

Pate, A.M., M cPherson, M., \& Silloway, G. (1987). T he M inneapolis Community Crime Prevention Experiment: draft evaluation report. Washington, DC : Police Foundation.

Pawson, R ., \& Tilley, N. (1997). Realistic evaluation. Londres: Sage.

Pease, K. (1991). The K irkholt Project: preventing burglary on a B ritish public housing estate. Security Journal, 2, 73-77.

Pease, K. (1992). Preventing burglary on a British housing estate. In. R.V. Clarke (ed.), Situational crime prevention: successful case studies (223229). N ew York: Harrow \& Heston.

Pettitt, A.N . (1980). A simple cumulative sum type statistic for the change-point problem with zero-one observations. Biometrika, 67 (1), 79-84.

Rosenbaum, D.P., Lewis, D.A., \& Grant, J.A. (1985). T he impact of community crime prevention programs in Chicago: can neighbourhood organization make a difference? Final Report, vol.1. Evanston: N orthwestern University Center for U rban Affairs and Policy Research.

Sherman, L.W., Gottfredson, D., Mackenzie, D., Eck, J., Reuter, P., \& Bushway, S. (1997). Preventing crime: what works, what doesn't, what's promising, Washington: U.S. D epartment of Justice.

Skogan, W. (1990). D isorder and decline: crime and the spiral of decay in A merican neighbourhoods. N ew York : Free Press.

Tilley, N. (1993). After Kirkholt? Theory, method and results of replication evaluations. Crime D etection and Prevention Series, 47. Londres: Home $O$ ffice.

Tilley, N ., Pease, K., H ough, M ., \& Brown, R. (1999). Burglary prevention: early lessons from the crime reduction programme. Crime Reduction Research Series Paper 1, Londres: H ome 0 ffice.

Tilley, N., \& Webb, J. (1994). Burglary reduction: findings from Safer Cities Schemes. Crime D etection and Prevention Series, 51. Londres: Home 0 ffice. 
ANNEXE 1

Données chronologiques des mesures de prévention et indicateurs

de répression à Boudhenville (octobre 1998 à dócembre 1999)

\begin{tabular}{|c|c|c|c|c|c|c|c|c|c|c|c|c|c|c|c|}
\hline & $\begin{array}{l}\stackrel{\infty}{\circ} \\
\stackrel{\circ}{\leftrightarrows} \\
\text { ঢ̆ }\end{array}$ & 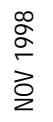 & $\begin{array}{l}\text { 号 } \\
\text { 岃 }\end{array}$ & 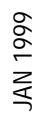 & 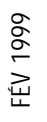 & 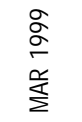 & 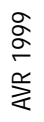 & 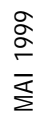 & $\begin{array}{l}\text { 오 } \\
\stackrel{ }{-1} \\
\text { 으 }\end{array}$ & $\begin{array}{l}\text { ㅇ } \\
\text { ㅁ } \\
\Xi\end{array}$ & $\begin{array}{l}\text { 오 } \\
\text { Оे } \\
\text { ㅇ } \\
\text { 운 }\end{array}$ & $\begin{array}{l}\text { ㅇ } \\
\text { Оे } \\
\text { 岀 }\end{array}$ & 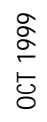 & $\begin{array}{l}\text { 음 } \\
\text { Оㄱㅁ } \\
\text { 을 }\end{array}$ & 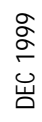 \\
\hline Cambriolages & 40 & 42 & 17 & 7 & 21 & 25 & 16 & 15 & 16 & 22 & 18 & 22 & 11 & 12 & 18 \\
\hline Mesures dinformation & - & - & - & - & - & 25119 & - & 16 & 40 & - & 79 & 50118 & 25 & 79 & - \\
\hline Mesures situationnelles & - & - & 16 & 79 & 8 & 32 & 20 & 31 & 32 & 79 & 50 & 31 & 80 & 40 & 39 \\
\hline Mses en acausation & 1 & 1 & 12 & 15 & 16 & o & 2 & 2 & o & 3 & 1 & 2 & 0 & 2 & 3 \\
\hline Nombre de résolutions & 6 & 10 & 4 & 2 & 12 & 2 & $\mathrm{o}$ & 5 & 1 & o & 1 & 2 & 1 & 5 & 9 \\
\hline
\end{tabular}

